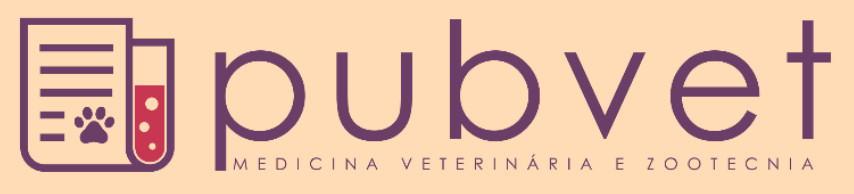

https://doi.org/10.31533/pubvet.v12n11a208.1-6

\title{
Condições higiênicossanitárias do caldo de cana de açúcar comercializado em Teresina - Piauí
}

\author{
Cícero Gilcélison da Silva $\operatorname{Xavier}^{1^{*}}$, Rafael Gomes Abreu Bacelar ${ }^{2} \bullet$, Eldo José \\ ${\text { Rodrigues dos } \operatorname{Santos}^{3} \odot \text {, Juliana Alexandre Ianiceli }}^{1 \bullet}$, Mariana Castro Brito ${ }^{1}{ }^{\ominus}$, Maria \\ Christina Sanches Muratori ${ }^{4} \bullet$
}

\author{
${ }^{1}$ Estudante de Medicina Veterinária da Universidade Federal do Piauí, Teresina-PI, Brasil. \\ ${ }^{2}$ Doutorando em Ciência Animal na Universidade Federal do Piauí, Teresina-PI, Brasil. \\ ${ }^{3}$ Residente em Medicina Veterinária na Universidade Federal do Piauí, Teresina-PI, Brasil. \\ ${ }^{4}$ Professora da Universidade Federal do Piauí, Departamento de Morfofisiologia Veterinária. Teresina-PI, Brasil. \\ *Autor para correspondência, E-mail: cicerogilcelison@gmail.com
}

RESUMO. O Brasil é um grande produtor de cana de açúcar, seu caldo é extraído por meio de moagem mecânica ou manual. Muitas vezes os manipuladores exercem outras funções concomitantemente, representando um dos meios de contaminação. Em vista disso, pode haver a contaminação por microrganismos e sujidades, desde o transporte e armazenamento até o seu consumo. Essa contaminação pode advir de condições inadequadas. Pelo exposto, objetivou-se avaliar as condições higiênicossanitárias do caldo de cana comercializado em Teresina, PI. As amostras foram obtidas em estabelecimentos escolhidos randomicamente. Foram coletadas nove amostras do caldo de cana em natureza, sendo três em cada estabelecimento e nove amostras industrializadas adquiridas em único estabelecimento, da mesma marca, sendo três amostras em cada dia, perfazendo um total de 18 amostras. Nas amostras em natureza houve a presença de coliformes a $35^{\circ} \mathrm{C}$ e a $45^{\circ} \mathrm{C}$ e ausência de Samonella spp em todas as amostras. Nas análises do caldo industrializado não foram encontrados coliformes a $35^{\circ} \mathrm{C}$ e a $45^{\circ} \mathrm{C}$, como também Salmonella spp em nenhuma das amostras. As condições higiênicossanitárias satisfatórias do caldo de cana comercializado em Teresina, PI, depende do estabelecimento e se o mesmo é industrializado.

Palavras chave: caldo industrializado, coliformes, Sacharium, Salmonella

\section{Hygienic and sanitary conditions of commercialized sugarcane juice in Teresina - Piauí}

ABSTRACT. Brazil is a major producer of sugar cane; its juice is extracted by means of mechanical or manual grinding. Often the manipulators perform other functions concomitantly, representing one of the means of contamination. In view of this, contamination by microorganisms and soils, from transport and storage to consumption, may result from inadequate conditions. The objective of this study was to evaluate the hygienic and sanitary conditions of cane juice marketed in Teresina, PI. Samples were obtained from randomly selected establishments. Nine samples of cane juice were collected in nature, three in each establishment and nine industrialized samples purchased in a single establishment, of the same brand, three samples each day, making a total of 18 samples. In the samples in nature, coliforms were present at $35^{\circ} \mathrm{C}$ and at $45^{\circ} \mathrm{C}$ and absence of Samonella spp in all samples. In the analyzes of the industrialized broth no coliforms were found at $35^{\circ} \mathrm{C}$ and at $45^{\circ} \mathrm{C}$, as well as Salmonella spp in none of the samples. The satisfactory sanitary and sanitary conditions of cane juice commercialized in Teresina, PI, depends on the establishment and if it is industrialized.

Keywords: coliforms, industrialized broth, Sacharium, Salmonella 


\title{
Condiciones higiénicas del caldo de caña de azúcar comercializado en Teresina - Piauí
}

\begin{abstract}
RESUMEN. Brasil es un gran productor de caña de azúcar, su caldo es extraído por medio de molienda mecánica o manual. Muchas veces los manipuladores ejercen otras funciones concomitantemente, representando uno de los medios de contaminación. En vista de ello, puede haber la contaminación por microorganismos y suciedad, desde el transporte y almacenamiento hasta su consumo, esa contaminación puede provenir de condiciones inadecuadas. Por lo expuesto, se objetivó evaluar las condiciones higiénicas del caldo de caña comercializado en Teresina, PI. Las muestras se obtuvieron en establecimientos seleccionados aleatoriamente. Se recogieron nueve muestras del caldo de caña in natura, siendo tres en cada establecimiento y nueve muestras industrializadas adquiridas en un único establecimiento, de la misma marca, siendo tres muestras cada día, con un total de 18 muestras. En las muestras in natura hubo la presencia de coliformes a $35^{\circ} \mathrm{C}$ ya $45^{\circ} \mathrm{C}$ y ausencia de Samonella spp en todas las muestras. En los análisis del caldo industrializado no se encontraron coliformes a $35^{\circ} \mathrm{C}$ ya $45^{\circ} \mathrm{C}$, como también Salmonella spp en ninguna de las muestras. Las condiciones higiénico-satisfactorias satisfactorias del caldo de caña comercializado en Teresina, PI, depende del establecimiento y si el mismo es industrializado.
\end{abstract}

Palabras clave: caldo industrializado, coliformes, Sacharium, Salmonella

\section{Introdução}

O caldo de cana, também conhecido como garapa, é uma bebida obtida a partir da moagem da cana de açúcar (Saccharum spp.), por um equipamento chamado moenda. É consumida in natura, possuindo um sabor agradável, refrescante e energética. Sua comercialização acontece em lanchonetes e feiras livres, representando riscos à saúde, por causa das condições higiênicossanitárias insatisfatórias durante a manipulação e acondicionamento da matéria prima (Carvalho et al., 2016; Norberg et al., 2012; Silva, 2010).

A RDC 218 de 29 de julho de 2005, define bebida preparada com vegetais: "substância ou mistura de substâncias obtida da polpa ou de outras partes de vegetais, acrescida ou não de outros ingredientes, destinada ao consumo, tais como água de coco, caldo de cana, polpas e salada de frutas, sucos de frutas e hortaliças, vitaminas ou batidas de frutas e similares" (Brasil, 2005).

O caldo de cana possui alta concentração de açúcares e baixa acidez, por isso é muito susceptível a deterioração principalmente por leveduras que levam à sua fermentação. Apesar disso, a RDC 12/2001 estabelece padrões microbiológicos apenas para coliformes $45^{\circ} \mathrm{C}$ e Salmonella spp (Brasil, 2001; Prati \& Camargo, 2008). A higienização no preparo visa além da preservação da palatabilidade e qualidade microbiológica dos alimentos, um produto que tenha qualidades nutricionais e sensoriais adequados não oferecendo riscos à saúde. Os vendedores/manipuladores do caldo de cana na maioria das vezes possui baixo conhecimento sobre condições higiênicossanitárias adequadas, alguns são vendedores ambulantes com pouca infraestrutura, constituindo um alto risco para a saúde dos consumidores (Felipe \& Miguel, 2012; Silva, 2010; Sprenger et al., 2016).

Objetivou-se fazer a contagem de coliformes $35^{\circ} \mathrm{C}$ e $45^{\circ} \mathrm{C}$, representados em número mais provável por miligramas $(\mathrm{NMP} / \mathrm{mL})$ e análise de Salmonella spp em caldos de cana comercializados em Teresina, PI.

\section{Material e métodos}

As amostras do caldo de cana de açúcar industrializado foram adquiridas em único estabelecimento, sendo todas da mesma marca. Foram coletadas três amostras por dia, durante três dias, totalizando nove unidades. Também foram coletadas amostras de vendedores ambulantes, onde foram adquiridas em três ambulantes diferentes, selecionados ao acaso. Onde coletavase uma amostra em cada vendedor por dia, durante três dias, perfazendo nove amostras, e juntando as amostras do estabelecimento com as coletadas dos ambulantes em um total de 18 amostras.

Os caldos de cana de açúcar industrializados foram adquiridos em embalagem própria do fabricante (copos de $300 \mathrm{~mL}$ ), envasadas 
assepticamente. Os caldos de cana de açúcar de vendedor ambulante foram obtidos em embalagens fornecidas pelo vendedor, copos de $200 \mathrm{~mL}$ com tampa, sendo fechadas imediatamente após o ato da compra.

Após a coleta, as amostras foram encaminhadas em recipiente isotérmico com gelo até o Laboratório de Controle Microbiológico de Alimentos do Núcleo de Estudos Pesquisas e Processamento de Alimentos (NUEPPA), localizado no Centro de Ciências Agrárias (CCA) da Universidade Federal do Piauí (UFPI), onde foram realizadas as análises microbiológicas.

Foram transferidas assepticamente alíquotas com $25 \mathrm{~mL}$ do caldo de cana de açúcar para um frasco com $225 \mathrm{~mL}$ contendo água peptonada a $0,1 \%$, formando a diluição inicial $\left(10^{-1}\right)$. A partir desta, preparadas diluições decimais seriadas até $10^{-3}$. Para o teste presuntivo de coliformes, foram inoculados $1 \mathrm{~mL}$ de cada diluição em uma série de três tubos com Caldo Lauryl Triptose (LST). Em seguida incubados à $37^{\circ} \mathrm{C}$, de 24 a 48 horas. Para o teste confirmativo de coliformes, dos tubos positivos no LST, foram repicados uma alça de platina para tubos com caldo Escherichia coli (Ec), incubados em banho-maria a $45,5^{\circ} \mathrm{C}$ por 24 horas e uma alça para o caldo verde brilhante (VB), em seguida incubados a $37^{\circ} \mathrm{C}$ por 24 horas. Analisou-se presença de turvação e gás no tubo de Duhran (APHA, 2014).

O número de testes positivos para cada diluição foi anotado. Após os resultados foram analisados conforme a técnica de Número Mais Provável (NMP). Na pesquisa de Salmonella spp. os frascos contendo a diluição $10^{-1}$ com água peptonada a $0,1 \%$ foram incubados a $37^{\circ} \mathrm{C}$ por 24 horas. Após alíquotas com $0,1 \mathrm{~mL}$ e $1,0 \mathrm{~mL}$ foram transferidos, respectivamente, para os caldos de enriquecimento seletivo Rappaport-Vassiliadis e Selenito-Cistina, e incubados a $37^{\circ} \mathrm{C}$ por 24 horas. Depois da incubação, os tubos que estavam turvados ou com alguma alteração, foram semeados em placas de Petri com agar Salmonella-Shigella e agar Hektoen em seguida incubadas por 24 horas a $37^{\circ} \mathrm{C}$ para posterior leitura (APHA, 2014).

$\mathrm{O}$ experimento foi desenvolvido em delineamento inteiramente casualizado com fatorial $4 \times 3$ (quatro estabelecimentos, três repetições), com três amostras representadas por amostras de $300 \mathrm{~mL}$ e $200 \mathrm{~mL}$. Os resultados quantitativos (coliformes $35^{\circ} \mathrm{C}$ e $45^{\circ} \mathrm{C}$ ) foram transformados em logaritmos para análise de variância e correlação pelo teste de Kruskal Wallis, com significância $\mathrm{P}<0,001$.

\section{Resultados e discussão}

Com base na análise microbiológica para contagem de coliformes a $35^{\circ} \mathrm{C}$ e a $45^{\circ} \mathrm{C}$, podese observar que não houve diferença $(\mathrm{P}>0,05)$ entre as amostras dos caldos de cana de açúcar comercializado em natureza (Tabela 1), apesar da amostra em natureza 1 ter contagem acima do estabelecido pela legislação. Todavia, houve diferença $(\mathrm{P}<0,05)$ entre as amostras do caldo de cana em natureza e as amostras industrializadas. Quanto a contaminação por Salmonella spp. não houve a presença em nenhuma das amostras.

Não houve diferença entre as amostras em natureza; porém a amostra em natureza 1 estava imprópria para o consumo devido à média $2,2 \pm$ $0,2 / \mathrm{mL}$ para coliformes a $45^{\circ} \mathrm{C}$ estar acima do estabelecido pela RDC 12/2001, representando 16,7\% das amostras (Brasil, 2001).

Moura et al. (2018) realizaram a avaliação microbiológica do caldo de cana de açúcar comercializado também no município de Teresina, PI, onde em sua análise todas as amostras apresentavam contagem de coliformes a $45^{\circ} \mathrm{C}$ acima do que preconiza a RDC 12/2001, diferindo das análises do presente trabalho, visto que somente a amostra em natureza 1 demonstrou-se fora do padrão (Brasil, 2001). Em estudos realizados por Carvalho et al. (2016), Prado et al. (2010) e Sprenger et al. (2016) foi encontrado coliformes $45^{\circ} \mathrm{C}$ em 40\%; 10,6\% e $30 \%$ respectivamente, das amostras analisadas.

As amostras em natureza 2 e 3 tiveram média $1,6 \pm 0,6 / \mathrm{mL}$ e $1,8 \pm 0,6 / \mathrm{mL}$, respectivamente, abaixo do padrão estabelecido pela RDC 12/2001; porém, merecendo atenção pois a multiplicação destes microrganismos pode ocorrer rapidamente quando em condições favoráveis ao crescimento. A presença destes micro-organismos sugere que a contaminação possa ter ocorrido na manipulação ou contaminação cruzada por meio de equipamentos e utensílios utilizados para a obtenção do caldo (Brasil, 2001; Prado et al., 2010).

Em observação direta no ato da compra das amostras em natureza, verificou-se $o$ acondicionamento da matéria prima sobre a moenda ou no chão dentro de sacos ráfia. $\mathrm{O}$ bagaço da cana era jogado no chão atrás da moenda. 
Tabela 1. Contagem de coliformes a $35^{\circ} \mathrm{C}$ e a $45^{\circ} \mathrm{C}$, representados em número mais provável por mililitros $(\mathrm{NMP} / \mathrm{mL})$ e análise de Salmonella spp. em caldos de cana comercializados em Teresina, PI.

\begin{tabular}{lccc}
\hline Amostras & Coliformes a $35^{\circ} \mathrm{C} / \mathrm{mL}$ & Coliformes a $45^{\circ} \mathrm{C} / \mathrm{mL}$ & Salmonella spp. em $25 \mathrm{~mL}$ \\
\hline Em natureza 1 & $2,4^{\mathrm{a}} \pm 0,2$ & $2,2^{\mathrm{a}} \pm 0,2$ & Ausência \\
Em natureza 2 & $2,4^{\mathrm{a}} \pm 0,3$ & $1,6^{\mathrm{a}} \pm 0,6$ & Ausência \\
Em natureza 3 & $2,2^{\mathrm{a}} \pm 0,6$ & $1,8^{\mathrm{a}} \pm 0,6$ & Ausência \\
Industrializada 1 & $0,00^{\mathrm{b}} \pm 0,0$ & $0,00^{\mathrm{b}} \pm 0,0$ & Ausência \\
Industrializada 2 & $0,00^{\mathrm{b}} \pm 0,0$ & $0,00^{\mathrm{b}} \pm 0,0$ & Ausência \\
Industrializada 3 & $0,00^{\mathrm{b}} \pm 0,0$ & $0,00^{\mathrm{b}} \pm 0,0$ & Ausência \\
\hline
\end{tabular}

a, b: letras iguais em coluna, resultados semelhantes $(\mathrm{P}<0,001)$. NMP/mL em $\log _{10}(\mathrm{x}+1)=$ número mais provável por mililitro, em logaritmos da base dez, acrescentados de uma unidade. Padrão: máximo 2,0 por $\mathrm{mL}$ para coliformes a $45^{\circ}$ e ausência em $25 \mathrm{~mL}$ para Salmonella spp. (Brasil, 2001).

O transporte da matéria prima sendo realizado ao ar livre exposta à contaminação. Os manipuladores realizavam outras atividades concomitantemente. Segundo as recomendações da RDC 218/2005, a matéria prima deve ser transportada sob condições que preservem sua integridade e qualidade sanitária; os equipamentos de moagem e de extração utilizados no preparo de alimentos e bebidas vegetais devem dispor de proteção contra pragas e vetores. Ainda, os manipuladores de alimentos devem ser capacitados em higiene pessoal, manipulação higiênica dos alimentos e doenças transmitidas por alimentos (Brasil, 2005).

Em pesquisa realizada recentemente por Sprenger and Risolia (2017) demonstraram um grave problema quando se trata na falha do transporte da cana de açúcar; conhecimento e treinamento dos manipuladores sobre higiene; proteção contra pragas e vetores; armazenamento adequado do lixo e limpeza e lavagem da cana. Dentre estes citados, um dado bastante expressivo é o fato de que em nenhum dos locais estudados os manipuladores tinham total conhecimento sobre higiene, assim como de proteções contra pragas e vetores. Por outro lado, identificaram que 93,6\% dos locais analisados a cana de açúcar era lavada e sanitizada. Reforçando assim, que a presença de sujidades e microrganismos podem advir dos manipuladores e instrumentos utilizados (Carvalho et al., 2016).

Quanto a presença de coliformes a $35^{\circ} \mathrm{C}$ a legislação não estabelece limites; porém, nas amostras em natureza 1,2 e 3 houve a presença com médias $2,4 \pm 0,2 / \mathrm{mL}, 2,4 \pm 0,3 / \mathrm{mL}, 2,2 \pm$ $0,6 / \mathrm{mL}$ respectivamente, sem diferença $(\mathrm{P}>0,5)$ entre as mesmas. Em estudo feito por Brezovsky et al. (2016), 20\% das amostras demonstraram-se impróprias ao consumo devido a contaminação na margem de $10^{5}$ e $40 \%$ das amostras na ordem de $10^{4}$, o que eles consideraram este dado preocupante visto que a cana de açúcar armazenada de forma incorreta pode ocasionar uma rápida multiplicação de microrganismos tornando-as impróprias para o consumo.

No presente trabalho não foi encontrada Salmonella spp. em nenhuma das amostras em natureza conforme preconiza a legislação, estando assim em consonância com os resultados obtidos por Carvalho et al. (2016), Moura et al. (2018) e Prado et al. (2010); porém, a bactéria foi encontrada em pesquisas realizada por Brezovsky et al. (2016).

Nas amostras industrializadas não foram encontrados coliformes a $35^{\circ} \mathrm{C}$ e a $45^{\circ} \mathrm{C}$, como também Salmonella spp. A ausência destes microrganismos pode ter sido garantida por meio do uso de algum tratamento térmico, como por exemplo a pasteurização, que realizada a $110^{\circ} \mathrm{C}$ por 10 s é o suficiente para garantir a ausência destes micro-organismos (Silva \& Faria, 2006).

Silva and Faria (2006) avaliaram a qualidade do caldo de cana de açúcar envasado a quente e por sistema asséptico e a partir de seus resultados concluíram que "o envase a quente mostrou-se mais adequado para o processamento do caldo de cana, tanto em termos sensoriais quanto em relação às alterações físico-químicas, ainda com a vantagem de ser um processo mais simples e acessível aos pequenos produtores", sendo considerado estéreis segundo a RDC 12/2001 (Brasil, 2001).

Em 60\% das enfermidades causadas por alimentos os manipuladores são responsáveis por $26 \%$ dos surtos, conforme Silva et al. (2013). Isso demostra que é preciso políticas a fim de capacitar estes no que diz respeito à segurança alimentar em todas as etapas de produção. A capacitação dos manipuladores é eficaz na ampliação do conhecimento destes, segundo dados da pesquisa realizada por Devides et al. (2014), onde 100\% 
dos manipuladores mostraram maior domínio após a realização da capacitação.

A contaminação encontrada na análise é reflexo da ausência de condições higiênicossanitárias em todas as etapas de produção, no transporte, armazenamento, manipulação e preparação. Políticas devem ser implementadas cada vez mais a fim de capacitar os manipuladores no que diz respeito a higiene de uma maneira geral, visto que estes representam uma questão fundamental na qualidade higiênicossanitárias.

\section{Conclusão}

Em relação à pesquisa de Salmonella spp. todas as amostras em natureza e industrializadas encontram-se apropriadas ao consumo. Porém, quanto à presença de coliformes a $45^{\circ} \mathrm{C}$ acima do padrão nas amostras em natureza tornando-as impróprias ao consumo, varia de acordo com o estabelecimento indicando condições higiênicossanitárias insatisfatórias. Ao passo que o caldo de cana industrializado possui melhores condições higiênicossanitárias quanto à ausência total de coliformes a $35^{\circ} \mathrm{C}$ e a $45^{\circ} \mathrm{C}$.

\section{Referências}

APHA. 2014. Standard methods for the examination of water and wastewater (Vol. 20): American Public Health Association.

BRASIL. 2001. Ministério da Saúde. Agência Nacional de Vigilância Sanitária. Resolução RDC n. ${ }^{\circ}$ 12, de 2 de janeiro de 2001. Regulamento técnico sobre padrões microbiológicos para alimentos. D. O. U. Seção 1, p. 45-53, Brasília, 10 jan.

Brasil, 2005. Ministério da Saúde. Agência Nacional de Vigilância Sanitária. Resolução RDC $n^{\circ} 218$, de 29 de julho de 2005. Regulamento técnico de procedimentos higiênico-sanitários para manipulação de alimentos e bebidas preparados com vegetais. D. O. U. Brasília, DF, 1 ago.

Brezovsky, F. R., Valiatti, T. B., Romão, N. F., Passoni, G. P., \& Sobral, F. d. O. S. 2016. Avaliação microbiológica e microscópica do caldo de cana comercializado em Ji-Paraná. Ensaios e Ciência, Ciências Biológicas, Agrárias e Saúde, 20(2), 111-115.

Carvalho, C. T., Santos, R. L. S., Araújo, L. B. A., \& Lima, J. P. S. 2016. Análise microbiológica do caldo de cana comercializado por ambulantes na cidade de Natal-RN. Revista Científica da Escola da Saúde, 5(1), 95-104.

Devides, G. G. G., Maffei, D. F., \& Catanozi, M. P. L. M. 2014. Perfil socioeconômico e profissional de manipuladores de alimentos e o impacto positivo de um curso de capacitação em boas práticas de fabricação. Brazilian Journal of Food Technology, 17, 166-176.

Felipe, L. M., \& Miguel, D. P. 2012. Análise da qualidade microbiológica do caldo de cana. FAZU em Revista, 8, 77-82.

Moura, L. B. D., Cavalcante, L. G. C., Nonato, R., Júnior, C. M., Leitão, J. M. S. R., Fernanda, E. \& Juliane, B. 2018. Avaliação microbiológica do caldo de cana comercializado em lanchonetes no centro de Teresina-Piauí. Revista Eletrônica Acervo Saúde, 14, S1684S1688.

Norberg, A. N., Oliveira, J. T. M., Monteiro, A. N., Sanches, F. G., Ribeiro, P. C., \& SerraFreire, N. M. 2012. Análise qualitativa e quantitativa de caldos de cana comercializados na região da Baixada Fluminense, estado do Rio de Janeiro, Brasil, quanto à poluição por Staphylococcus aureus. Revista de Ciência $e$ Tecnologia, 12(2), 78-86.

Prado, S. d. P. T., Bergamini, A. M. M., Ribeiro, E. G. A., Castro, M. d. C. S., \& Oliveira, M. A. 2010. Avaliação do perfil microbiológico e microscópico do caldo de cana in natura comercializado por ambulantes. Revista do Instituto Adolfo Lutz, 69(1), 55-61.

Prati, P., \& Camargo, G. A. 2008. Característica do caldo de cana e sua influencia na estabilidade da bebida. BioEng, Campinas-SP, 2(1), 37-44.

Silva, A. S. 2010. Avaliação microbiológica do caldo de cana comercializado na orla marítima da cidade de Salvados-Bahia. Revista Virtual, 6(2), 74-85.

Silva, G. R., Barros, M. L. G., Barbosa, M. V. F., Siqueira, M. G. F. M., Oliveira, A. E., Lins, L. F., \& Moura, A. P. B. L. 2013. Percepção do conceito de higiene e segurança alimentar dos manipuladores de produtos cárneos de mercado público, Recife-PE, Brasil. Acta Veterinária Brasílica, 7(2), 158-163.

Silva, K. S., \& Faria, J. A. F. 2006. Avaliação da qualidade de caldo de cana envasado a quente e por sistema asséptico. Ciência e Tecnologia de Alimentos, 26(4), 754-758. 
Sprenger, L. K., \& Risolia, L. W. 2017. Condições higiênicas sanitárias da produção do caldo de cana em Curitiba, Paraná. Archives of Veterinary Science, 22(2), 1-7.

Sprenger, L. K., Risolia, L. W., Hamdar, S. Z., \& Molento, M. B. 2016. Análise microbiológica de caldos de cana comercializados em Curitiba, Paraná. Archives of Veterinary Science, 21(4), 1-7.
Recebido: 6 Agosto 2018 .

Aprovado:1 Setembro 2018.

Publicado: 20 Novembro 2018.

Licenciamento: Este artigo é publicado na modalidade Acesso Aberto sob a licença Creative Commons Atribuição 4.0 (CC-BY 4.0), a qual permite uso irrestrito, distribuição, reprodução em qualquer meio, desde que o autor e a fonte sejam devidamente creditados 